\title{
Evidências científicas sobre a suplementação de vitamina D em pacientes acometidos por COVID-19: uma revisão bibliográfica integrativa
}

\author{
Scientific evidence on vitamin D supplementation in patients affected by COVID-19: an integrative
} bibliographic review

Evidencia científica sobre la suplementación de vitamina D en pacientes afectados por COVID-19: una revisión bibliográfica integrativa

\section{Resumo}

O objetivo dessa revisão foi avaliar os efeitos da suplementação de vitamina D em pacientes acometidos por COVID19. Na metodologia, foi realizado um levantamento por meio de busca eletrônica nos bancos de dados LILACS, PubMed e Cochrane, por artigos publicados em 2020 e 2021. Na pesquisa eletrônica inicial foi identificado 241 artigos na base PubMed, 68 artigos na Cochrane e 3 artigos na LILACS sobre o tema. Na primeira triagem, 187 estudos foram selecionados e 125 foram excluídos por não apresentarem as palavras "covid" e "vitamina D" no título e/ou resumo. Na segunda triagem, 177 artigos foram excluídos e 10 estudos foram selecionados para inclusão na revisão. Como resultado, 4 dos 10 incluídos envolveram pacientes com COVID-19 leve, enquanto 2 envolveram pacientes em estado grave. Em relação à idade, a maioria dos estudos envolvem pacientes com idade avançada e com comorbidades, como diabetes e hipertensão. Como conclusão, a suplementação de vitamina D é mais eficaz em pacientes com COVID-19 de leve a moderada, principalmente em pacientes que já faziam suplementação da vitamina antes de serem acometidos pela doença. Sobre os pacientes com COVID-19 grave, as evidências mostraram que altas doses podem gerar efeitos rápidos e satisfatórios no tempo de internação, porém o início tardio da suplementação pode ser um fator limitador.

Palavras-chave: COVID-19; Vitamina D; Suplementação.

\begin{abstract}
The objective of this review was to assess the effects of vitamin D supplementation in patients affected by COVID-19. In the methodology, a survey was carried out by means of an electronic search in data banks LILACS, PubMed and Cochrane, by articles published in 2020 and 2021. In the initial electronic search, 241 articles were identified in the PubMed base, 68 articles in Cochrane and 3 articles in LILACS on the subject. In the first screening, 187 studies were selected and 125 were excluded by not presenting the words "covid" and "vitamin D", not title and / or summary. In the second screening, 177 articles were excluded and 10 studies were selected for inclusion in the review. As a result, 4 two 10 included patients with mild COVID-19, while 2 involved patients in serious condition. In relation to life, a majority of two studies involve patients with advanced eating and comorbidities, such as diabetes and hypertension. As a conclusion, vitamin D supplementation is most effective in mild to moderate COVID-19 patients, mainly in patients who had vitamin supplementation before being treated for the disease. Regarding patients with severe COVID-19, the events will show that high doses can generate rapid and satisfactory effects, without time of hospitalization, therefore, or late start of supplementation can be a limiting factor.
\end{abstract}

Keyworks: COVID-19; Vitamin D; Supplementation.

\section{Resumen}

El objetivo de esta revisión fue evaluar los efectos de la suplementación con vitamina $\mathrm{D}$ en pacientes afectados por COVID-19. En la metodología, se realizó una encuesta mediante búsqueda electrónica en las bases de datos LILACS, PubMed y Cochrane, para artículos publicados en 2020 y 2021 En la búsqueda electrónica inicial se identificaron 241 artículos en la base de datos PubMed, 68 artículos en Cochrane y 3 artículos en LILACS sobre el tema. En la primera selección, se seleccionaron 187 estudios y se excluyeron 125 por no tener las palabras "covid" y "vitamina D" en el título y / o resumen. En la segunda selección, se excluyeron 177 artículos y se seleccionaron 10 estudios para su inclusión 
en la revisión. Como resultado, 4 de los 10 incluyeron pacientes involucrados con COVID-19 leve, mientras que 2 involucraron pacientes en una condición severa. En cuanto a la edad, la mayoría de los estudios involucran a pacientes con edad avanzada y comorbilidades como diabetes e hipertensión. En conclusión, la suplementación con vitamina D es más eficaz en pacientes con COVID-19 de un nivel moderado a moderado, especialmente en pacientes que ya estaban tomando suplementos de vitamina D antes de verse afectados por la enfermedad. En cuanto a los pacientes con COVID19 grave, la evidencia ha demostrado que las dosis altas pueden generar efectos rápidos y satisfactorios sobre la duración de la estancia, pero el inicio tardío de la suplementación puede ser un factor limitante.

Palabras clave: COVID-19; Vitamina D; Suplementación.

\section{Introdução}

O COVID-19 é uma doença caracterizada por uma síndrome respiratória aguda provocada pelo coronavírus 2 (SARSCoV-2), que causou impacto negativo em todo o mundo. O coronavírus 2 é o terceiro coronavírus humano a causar doença nas últimas duas décadas. Antes do surgimento do SARS-CoV-2, a Síndrome do Coronavírus Respiratório Aguda Grave (SARSCoV) apareceu em Guangdong, China, em 2002, causando mais de 8.000 infecções e 774 mortes em 37 países (Hetta et al., 2021).

A pandemia do COVID-19 representa uma ameaça sem precedentes à saúde humana, aos sistemas de saúde, à vida pública e à economia em todo o mundo. Isso é potencializado pelo falo das terapias eficazes para o curso de doenças graves ainda serem limitadas, visto que pouco se sabe sobre o vírus. No entanto, uma grande parte da população mundial sofre de deficiência de vitamina D, e a prevalência é particularmente alta entre pessoas com alto risco de COVID-19 (Brenner et al., 2021).

A deficiência de vitamina D é considerada um problema de saúde global que provoca muitas doenças, uma delas envolve as infecções virais no geral. No entanto, o mecanismo que descreve a relação dos baixos níveis de vitamina D com as infecções virais ainda não é totalmente compreendido, porém alguns podem estar relacionados com a imunorregulação e o potencial antiinflamatório, além de fatores celulares e virais que afetam a replicação do vírus (Hetta et al., 2021).

Nesse contexto, surge a vitamina D como uma alternativa de terapia adjuvante na COVID-19. A vitamina D é caracterizada por ser um hormônio esteroide lipossolúvel presente em fontes alimentares como peixes, fígados e ovos, e sendo metabolizada nas formas de vitamina D2 (ergocalciferol) e vitamina D3 (colecalciferol). Ambas se diferem pelo fato da vitamina D3 apresentar uma meia vida maior que a vitamina D2 e, devido a isso, está presente em maior concentração na circulação sanguínea (Pinheiro et al., 2020).

A vitamina D tem função conhecida no metabolismo de cálcio e fosfato no organismo, contribuindo para a manutenção da densidade óssea. Além disso, foram observados efeitos da vitamina D na atividade de células do sistema imune, e que níveis baixos de vitamina D estão associados com aumento nos riscos de desenvolvimento e agravamento de doenças como psoríase, Diabetes tipo 1, tuberculose e infecções respiratórias (Moreira et al., 2020).

De acordo com Zhou et al. (2019), os efeitos protetores da vitamina D também foram confirmados em infecções por pneumonia, tempestade de citocinas e síndrome da doença respiratória aguda (SDRA), que são fatores diretamente associados à infecção pelo coronavírus, sendo presente em estados grave da doença.

Dessa forma, é notória a importância de mais pesquisas em torno da suplementação de vitamina D como terapia adjuvante no tratamento de pacientes com COVID-19, uma vez que a tendência atual é a convivência com o coronavírus pelos próximos anos. Além disso, destaca-se a importância do estabelecimento da melhor dosagem para a prevenção e tratamento do COVID-19 e a possibilidade do desenvolvimento de dietas que aumentam gradativamente os níveis da vitamina, enfatizando a importância da presença do profissional nutricionista também no período pós pandemia.

Com isso, o objetivo dessa revisão bibliográfica foi trazer evidências sobre os efeitos da suplementação de vitamina D em pacientes acometidos por COVID-19. 


\section{Metodologia}

O presente estudo se trata de uma revisão bibliográfica integrativa, de natureza qualitativa e quantitativa, com abordagem exploratória que procura investigar a influência da suplementação de Vitamina D em pacientes acometidos por COVID-19 (Estrela, 2018). Sendo assim, foi realizado um levantamento por meio de busca eletrônica nos bancos de dados LILACS (Literatura Latino-Americana e do Caribe em Ciências da Saúde), PubMed e Cochrane, por artigos publicados em 2020 e 2021.

Para isso, utilizou-se as palavras-chave referentes à vitamina $\mathrm{D}$, à suplementação vitamínica e à covid, definidas através da estratégia PICO (acrônimo para P: população/pacientes; I: intervenção; C: comparação/controle; O: desfecho/outcome) e, em seguida, foram estabelecidos seus descritores conforme consulta aos Descritores em Ciências da Saúde (DeCS) e ao Medical Subject Headings (MeSH). Os termos encontrados foram cruzados entre si por meio do uso do operador booleano "OR" e "AND", a fim de restringir a pesquisa aos trabalhos que apresentavam ao mesmo tempo os termos selecionados. A Tabela 1 apresenta a estratégia de busca dos artigos científicos, relacionando as bases consultadas com os respectivos descritores e operadores.

Tabela 1: Estratégia de busca de artigos encontrados nas bases de dados.

\begin{tabular}{cc}
\hline Palavras-chave & Descritores \\
\hline Vitamina D & $\begin{array}{c}\text { (Calcifediol OR vitamin D OR Cholecalciferol OR } \\
\text { Hydroxycholecalciferols OR Ergocalciferols OR 25- } \\
\text { Hydroxyvitamin D 2 OR Dihydrotachysterol) }\end{array}$ \\
\hline Suplementação & (Supplementation) \\
\hline Covid & (Covid OR COVID-19 Virus Disease OR 2019 nCoV Infection OR \\
& COVID-19 Virus Infection OR SARS-CoV-2 Infection COVID-19 \\
Pandemic OR SARS Coronavirus 2 Infection)
\end{tabular}

Fonte: Dados da pesquisa (2021).

Na tabela 1 acima, é importante observar as palavras-chave que foram utilizadas para o desenvolvimento dos seus respectivos descritores, utilizados de forma associada na busca pelas bases científicas.

A seleção dos artigos foi dividida em triagens, e que na primeira foram selecionados artigos que apresentavam os termos "covid" e "vitamina D" no título ou resumo. Após a seleção dos artigos da primeira triagem, foi realizada uma segunda triagem, na qual foram utilizados critérios de inclusão e exclusão para a seleção dos artigos. Como critérios de inclusão, foram selecionados apenas estudos que se tratava de ensaios clínicos, casos clínicos, estudos que apresentassem o efeito da suplementação de vitamina D em paciente com COVID-19 e estudos que tivessem disponíveis na íntegra.

Para este estudo, foram excluídas as revisões bibliográficas, capítulos de livros, diretrizes, meta-análises, estudos de coorte, protocolos, comentários, resposta a autores, carta ao editor, estudos em andamento, estudos que não focalizavam nos resultados do efeito da suplementação de vitamina D em pacientes com COVID-19, estudos que não tinham o resumo disponível e artigos duplicados.

Por fim, todos os estudos que atenderam os critérios definidos foram recuperados na íntegra e analisados para inclusão na revisão. 


\section{Resultados e Discussão}

Na pesquisa eletrônica inicial foi identificado 241 artigos na base PubMed, 68 artigos na Cochrane e 3 artigos na LILACS sobre o tema "Avaliação do efeito da suplementação de vitamina D em pacientes acometidos por COVID-19" por um período entre 2019 e 2021 (Figura 1). Na primeira triagem, 187 estudos foram selecionados e 125 foram excluídos por não apresentarem as palavras "covid" e "vitamina D" no título e/ou resumo. Na segunda triagem, 177 artigos foram excluídos, dos quais 91 eram revisões, 3 não estavam disponíveis na íntegra, 7 se tratava de protocolo, 21 não abordavam a suplementação de vitamina D em pacientes com COVID-19, 6 eram respostas a autores, 5 eram comentários, 12 eram artigos duplicados, 1 era estudo de coorte, 1 era uma carta ao editor, 2 eram diretrizes, 25 estavam em andamento e 1 era uma meta-análise.

Figura 1: Fluxograma de estratégia de busca para seleção dos artigos incluídos na revisão.

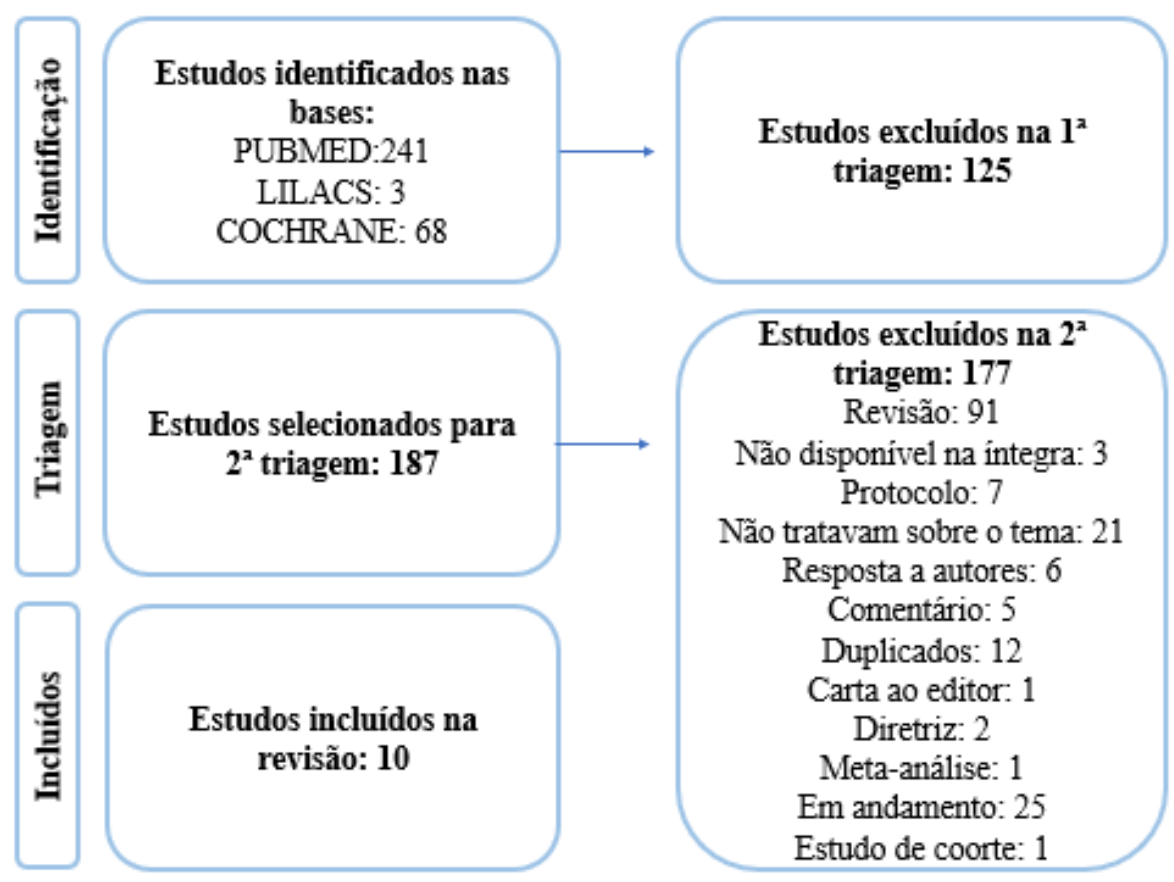

Fonte: Dados da pesquisa (2021).

Na figura acima, é importante observar que, após a aplicação dos descritores em cada uma das plataformas de busca citadas, os critérios previamente definidos para a inclusão e exclusão de artigos para o presente estudo foram cumpridos, resultando na inclusão de 10 artigos que possuem relação com o tema e que atendem os parâmetros que foram definidos pelos autores.

Após a segunda triagem, selecionou-se 10 trabalhos para incluir na revisão (Tabela 1), dos quais 4 estudos envolveram pacientes com COVID-19 leve, sendo eles Sabico et al. (2021), Lakkireddy et al. (2021), Rastogi et al., (2020) e Annweiler et al. (2020b); enquanto 2 envolveram pacientes em estado grave, sendo eles Murai et al. (2020) e Notz et al. (2021). Em relação à idade, a maioria dos estudos envolvem pacientes com idade avançada e com comorbidades, como diabetes e hipertensão, relatados nos estudos de Sabico et al. (2021), Lakkireddy et al. (2021), Murai et al. (2020), Annweiler et al. (2020a) e Ohaegbulam et al. (2020).

Em relação ao nível de vitamina D dos pacientes, os estudos de Ohaegbulam et al. (2020), Annweiler et al. (2020a), Sabico et al. (2021), Lakkireddy et al. (2021), Rastogi et al., (2020), Murai et al. (2020), Cereda et al. (2021) e Notz et al. (2021) 
relataram pacientes com deficiência da vitamina. No estudo de Annweiler et al. (2021), 2 pacientes foram admitidos no hospital com níveis ausentes de $25(\mathrm{OH}) \mathrm{D}$, mas o estudo não deixa explícito se ambos foram incluídos no ensaio.

Os resultados foram distribuídos em tabelas, sendo a Tabela 2 destinada aos estudos que relataram uso de dose padrão isolada de Vitamina D ou associada a doses altas e a Tabela 3 aos estudos que relataram uso de dose padrão isolada de Vitamina D ou associada a doses altas.

Tabela 2: Estudos que relataram uso de dose padrão isolada de Vitamina D ou associada a doses altas.

\begin{tabular}{|c|c|c|c|c|c|c|}
\hline AUTOR & LOCAL & $\begin{array}{c}\text { DIAS DE } \\
\text { ACOMPANH } \\
\text { AMENTO }\end{array}$ & $\begin{array}{l}\text { NÚMER } \\
\text { O DE } \\
\text { PACIEN } \\
\text { TES }\end{array}$ & $\begin{array}{c}\text { FAIXA } \\
\text { ETÁRIA }\end{array}$ & DOSAGEM & RESULTADOS \\
\hline $\begin{array}{l}\text { SABICO et al., } \\
2021\end{array}$ & $\begin{array}{l}\text { Reino } \\
\text { Unido da } \\
\text { Arábia } \\
\text { Saudita }\end{array}$ & 14 dias & 69 & 20 a 75 anos & $\begin{array}{l}\text { Vitamina D3 5000UI e } \\
\text { 1000UI durante } 2 \text { semanas } \\
\text { por via oral. }\end{array}$ & $\begin{array}{l}\text { Aumento significativo nos níveis } \\
\text { séricos de } 25(\mathrm{OH}) \mathrm{D} \text { apenas no } \\
\text { grupo de } 5000 \mathrm{UI} \text {; sobrevivência de } \\
\text { Kaplan-Meier revelou que o grupo } \\
\text { de } 5.000 \text { IU teve um tempo } \\
\text { significativamente menor para } \\
\text { recuperação (dias) do que o grupo de } \\
1.000 \text { IU na resolução da tosse. }\end{array}$ \\
\hline $\begin{array}{l}\text { OHAEGBULA } \\
\text { M } \text { et al., } 2020\end{array}$ & Nova York & 9 a 14 dias & 4 & 41 a 74 anos & $\begin{array}{l}\text { Colecalciferol: } \\
\text { diária de } 1000 \text { UI (dose } \\
\text { padrão); ou ergocalciferol: } \\
\text { Dose de } 50.000 \text { UI } \\
\text { diariamente por } 5 \text { dias } \\
\text { (dose alta). }\end{array}$ & $\begin{array}{l}\text { Os pacientes que receberam uma alta } \\
\text { dose de suplementação de vitamina } \\
\mathrm{D} \text { alcançaram a normalização dos } \\
\text { níveis de vitamina D e melhoraram a } \\
\text { recuperação clínica evidenciada por } \\
\text { períodos mais curtos de internação, } \\
\text { menores necessidades de oxigênio e } \\
\text { uma redução no status do marcador } \\
\text { inflamatório. }\end{array}$ \\
\hline $\begin{array}{l}\text { ANNWEILER } e t \\
a l ., 2021 .\end{array}$ & França & 3 meses & 95 & $88.0 \pm 5.5$ & $\begin{array}{l}\text { Suplementação diária de } \\
800 \text { UI por via oral; } \\
\text { em bólus: } 50.000 \text { UI por } \\
\text { mês, ou } 80.000 \text { UI ou } \\
100.000 \text { UI ou } 200.000 \text { UI } \\
\text { a cada } 2-3 \text { meses por via } \\
\text { oral. }\end{array}$ & $\begin{array}{l}76,1 \%(\mathrm{n}=51) \text { dos participantes } \\
\text { sobreviveram aos } 3 \text { meses no grupo } \\
\text { Intervenção, em comparação com } \\
\text { apenas } 53,6 \%(\mathrm{n}=15) \text { no grupo } \\
\text { Comparador. O grupo de } \\
\text { intervenção também teve maior } \\
\text { tempo de sobrevivência. }\end{array}$ \\
\hline
\end{tabular}

Fonte: Dados da pesquisa (2021).

Tabela 3: Estudos que relataram uso de alta dose de Vitamina D. 


\begin{tabular}{|c|c|c|c|c|c|c|}
\hline AUTOR & LOCAL & $\begin{array}{l}\text { DIAS DE } \\
\text { ACOMPA } \\
\text { NHAMEN } \\
\text { TO }\end{array}$ & PACIENTES & $\begin{array}{c}\text { FAIXA } \\
\text { ETÁRIA }\end{array}$ & DOSAGEM & RESULTADOS \\
\hline $\begin{array}{l}\text { LAKKIRE } \\
\text { DDY et al., } \\
\quad 2021\end{array}$ & Índia & 8 ou 10 dias & 87 & $\begin{array}{c}45 \pm 13 \\
\text { anos }\end{array}$ & $\begin{array}{l}\text { Grupo experimental: } 60.000 \\
\text { UI de vit.D na forma de } \\
\text { aquoso nano solução por dia } \\
\text { durante } 8 \text { dias; Grupo } \\
\text { controle: tratamento padrão } \\
\text { para COVID-19. }\end{array}$ & $\begin{array}{l}\text { Grupo experimental: redução dos } \\
\text { marcadores inflamatórios e melhora do } \\
\text { nível sérico de vit.D para } 80-100 \mathrm{ng} / \mathrm{ml} \text {. }\end{array}$ \\
\hline $\begin{array}{l}\text { RASTOGI } \\
\text { et al., } 2020\end{array}$ & Índia & 7 a 14 dias & 40 & $\begin{array}{l}\text { Intervençã } \\
\text { o: } 50,0 \\
\text { Controle: } \\
\quad 47,5\end{array}$ & $\begin{array}{l}60.000 \text { UI de colecalciferol } \\
\text { diariamente ( } 5 \text { ml de solução } \\
\text { oral na forma de nano gotas) } \\
\text { por } 7 \text { dias }\end{array}$ & $\begin{array}{l}\text { Indivíduos com deficiência de vitamina D } \\
\text { tornaram o RNA SARS-CoV-2 negativo e } \\
\text { uma diminuição significativa no } \\
\text { fibrinogênio com suplementação de } \\
\text { colecalciferol em altas doses. }\end{array}$ \\
\hline $\begin{array}{l}\text { MURAI et } \\
\text { al., } 2021\end{array}$ & $\begin{array}{l}\text { São Paulo } \\
\text { (Brasil) }\end{array}$ & 1,4 dias & 240 & 56,2 anos & $\begin{array}{l}\text { Dose oral única de } 200.000 \\
\text { UI de vitamina D3 }(n= \\
\text { 120) ou placebo }(n=120) \text {. }\end{array}$ & $\begin{array}{l}\text { Dose alta não reduziu significativamente o } \\
\text { tempo de internação hospitalar. Os } \\
\text { resultados não apoiam o uso de uma dose } \\
\text { elevada de vitamina D3 para o tratamento } \\
\text { de COVID-19 moderado a grave. }\end{array}$ \\
\hline $\begin{array}{l}\text { NOTZ et } \\
\text { al., } 2021\end{array}$ & Alemanha & 10 a 15 dias & 26 & 59,5 anos & $\begin{array}{l}200.000 \text { UI de vitamina D3 } \\
\text { como dose de ataque e } \\
10.000 \text { UI diariamente por } \\
\text { via enteral. }\end{array}$ & $\begin{array}{l}\text { Após } 10 \text { a } 15 \text { dias, os parâmetros clínicos } \\
\text { não diferiram entre pacientes com níveis } \\
\text { suficientes e insuficientes ou deficientes } \\
\text { de } 25 \text {-hidroxivitamina } D \text {, respectivamente. }\end{array}$ \\
\hline $\begin{array}{l}\text { ANNWEIL } \\
\text { ER } \text { et al., } \\
\text { 2020b }\end{array}$ & França & $\begin{array}{l}36 \pm 17 \\
\text { dias. }\end{array}$ & 66 & $\begin{array}{l}87,7 \pm 9,0 \\
\text { anos }\end{array}$ & $\begin{array}{l}\text { Grupo intervenção: bolus } \\
\text { oral de } 80.000 \text { UI de } \\
\text { vitamina D3 após início dos } \\
\text { sintomas; } \\
\text { Grupo comparador: } \\
\text { nenhuma suplementação } \\
\text { recente. }\end{array}$ & $\begin{array}{l}82,5 \% \text { dos participantes do grupo de } \\
\text { intervenção sobreviveram ao COVID-19, } \\
\text { em comparação com apenas } 44,4 \% \text { no } \\
\text { grupo comparador. }\end{array}$ \\
\hline $\begin{array}{l}\text { ANNWEIL } \\
\text { ER } \text { et al., } \\
\text { 2020a }\end{array}$ & França & 14 dias & 77 & $\begin{array}{l}88 \pm 5 \\
\text { anos }\end{array}$ & $\begin{array}{l}\text { Grupo 1: doses de } 50.000 \\
\text { UI de vitamina D3 por mês, } \\
\text { ou as doses de } 80.000 \text { UI ou } \\
\text { 100.000 UI de vitamina D3 } \\
\text { a cada 2-3 meses (oral) } \\
\text { Grupo 2: } 80.000 \text { UI de } \\
\text { vitamina D3 (oral) }\end{array}$ & $\begin{array}{l}\text { O Grupo } 1 \text { teve maior tempo de } \\
\text { sobrevivência do que o Grupo 3, que não } \\
\text { recebeu suplementação. } \\
\text { A suplementação regular de vitamina D } \\
\text { em bolus foi associada a COVID-19 } \\
\text { menos grave e melhor sobrevida em } \\
\text { idosos frágeis. }\end{array}$ \\
\hline $\begin{array}{l}\text { CEREDA } \\
\text { et al., } 2021\end{array}$ & Itália & $\begin{array}{l}\text { Não } \\
\text { informado }\end{array}$ & 324 & $\begin{array}{l}\text { Não } \\
\text { informado }\end{array}$ & $\begin{array}{l}54.000 \text { UI / mês (> } 1800 \\
\text { UI/d). }\end{array}$ & $\begin{array}{l}\text { A suplementação de } 25 \mathrm{OHD} \text { não foi } \\
\text { associada à hospitalização, mas pareceu } \\
\text { ser um fator de risco para maior } \\
\text { mortalidade hospitalar no COVID-19. }\end{array}$ \\
\hline
\end{tabular}

Fonte: Dados da pesquisa (2021).

Nas Tabelas 2 e 3 acima, pode-se observar de importante o resumo dos estudos incluídos no trabalho, contendo o autor, o local onde foi realizado o estudo, a quantidade de dias de acompanhamento, a quantidade de pacientes, a faixa etária, a dosagem de vitamina D utilizada em cada estudo e os resultados alcançados. 
O Institute Of Medicine (IOM) recomenda uma necessidade média de vitamina D estimada de $10 \mu \mathrm{g} / \mathrm{d}$, o que equivale a 400 UI para todas as faixas etárias. Já a ingestão diária recomendada para indivíduos adultos até 70 anos de idade é de $15 \mu \mathrm{g} / \mathrm{d}$, equivalente a $600 \mathrm{UI}$; e para pessoas idosas com idade superior a 70 anos, é recomendado uma ingestão de $20 \mu \mathrm{g} / \mathrm{d}$, o que equivale a 800 UI. Em relação ao nível de ingestão superior tolerável de vitamina D, baseia-se em $100 \mu \mathrm{g} / \mathrm{d}$, o que equivale a 4.000 UI (Alburqueque, 2021).

Como proposto, em pessoas saudáveis a recomendação de vitamina D é entre 5 e $20 \mu \mathrm{g}$, o que equivale a 200 a 800 UI. Já em pacientes com diagnóstico de COVID-19, tem sido proposta a suplementação da vitamina para minimizar o agravamento da doença, porém ainda não é definido o limite de dosagem capaz de promover a regressão ou prevenção da doença (Rhodes $e t$ al., 2020).

No estudo de Ohaegbulam et al., (2020), foram analisados 4 pacientes com COVID-19 e hipovitaminose D, que receberam colecalciferol de 1000 UI diariamente (dose padrão) ou ergocalciferol 50.000 UI diariamente por 5 dias (dose alta) como parte da suplementação. No ensaio, os pacientes que receberam uma alta dose de suplementação de vitamina $\mathrm{D}$ alcançaram a normalização dos níveis de vitamina D e melhoraram a recuperação clínica evidenciada por períodos mais curtos de internação, em comparação com os pacientes que receberam a dose padrão.

Outro estudo no qual os pacientes também fizeram o uso de doses mais baixas de vitamina D, foi o de Sabico et al., (2021), em que foi observado que 5.000 UI de vitamina D3 oral ingeridos diariamente por 2 semanas reduziu substancialmente os dias de recuperação de tosse e ageusia, e isso foi clinicamente significativo em comparação com aqueles que tomaram a dose padrão de 1000UI. Vale ressaltar que os níveis circulantes de $25(\mathrm{OH}) \mathrm{D}$ de quase todos os participantes no início do estudo estavam na faixa de insuficiência ou deficiência leve, e que 5000 UI de vitamina D3 administrada por 2 semanas é seguro e tolerável, apesar da dose de segurança superior aceitável ser de 4000 UI (Rizzoli, 2020).

Esse estudo mostrou que a suplementação de vitamina D é importante mesmo em casos de COVID-19 de leve a moderado, principalmente em paciente com comorbidades, evitando o desencadeamento da forma mais grave da doença, uma vez que a vitamina $\mathrm{D}$ pode ter efeitos benéficos ao longo do tempo nas comorbidades que são fatores de risco para formas graves de COVID-19, como diabetes mellitus e hipertensão, que foram doenças apresentadas pelos pacientes tratados no estudo de Sabico et al., (2021) (Annweiler et al., 2021).

Além disso, é possível observar que a dose padrão de 1000UI de Vitamina D não promove alterações significativas no quadro do paciente com COVID-19, sendo insuficiente comparada às doses mais altas. Isso pode ocorrer por influência da faixa etária uma vez que em pacientes adultos mais velhos pode ter uma resposta mais baixa, além da deficiência de vitamina $\mathrm{D}$, que requer um período de suplementação muito mais longo ou uma dose mais alta para atingir um nível suficiente num curto período. Ademais, o quadro inflamatório causado pelo COVID-19 pode dificultar o aumento rápido dos níveis de vitamina D (Rhodes et al., 2020).

É válido destacar também que as baixas concentrações de vitamina D estão diretamente relacionadas ao aumento de citocinas inflamatórias em pacientes idosos, fumantes, obesos e com doenças crônicas não-transmissíveis (DCNT) como diabetes e hipertensão, causando um maior risco de mortalidade e dificultando o aumento dos níveis da vitamina (Rhodes et al., 2020).

No estudo de Lakkireddy et al., (2021), também participaram pacientes com COVID-19 leve a moderada com hipovitaminose D, porém não geriátricos como no estudo de Sabico et al., (2021). No entanto, nesse ensaio os participantes receberam uma dose alta de vitamina $\mathrm{D}$, ou seja, 60.000 UI na forma de aquoso por 8 ou 10 dias, dependendo do índice de massa corporal, junto com o tratamento padrão para COVID-19. O resultado também foi satisfatório, visto que o grupo experimental apresentou redução dos marcadores inflamatórios e melhora do nível sérico de vitamina $\mathrm{D}$, apesar da diferença na média de permanência hospitalar entre os grupos não ter sido significativa. $\mathrm{O}$ tratamento com alta dosagem não apresentou nenhuma reação adversa atribuível à toxicidade vitamina $\mathrm{D}$. 
Com isso, é possível observar que tanto os pacientes com hipovitaminose D e COVID-19 leve, que receberam dose alta e dose padrão de vitamina $\mathrm{D}$, apresentaram resultados semelhantes e satisfatórios, levantando a hipótese do potencial da vitamina D na recuperação de pacientes acometidos pela doença leve ou moderada. Ademais, é notória a rápida recuperação quando ocorre o uso de alta dosagem de vitamina $\mathrm{D}$, uma vez que é capaz de aumentar rapidamente os níveis de 25(OH)D sérico (Bruyere $e t$ al., 2015)

Outro estudo no qual foi usada a dosagem de 60.000UI de Vitamina D por um período curto, foi o de Rastogi et al. (2020). Esse estudo também foi realizado com indivíduos assintomáticos ou doença leve que apresentavam deficiência de vitamina D. Os resultados desse estudo também se mostraram satisfatórios, pois a dose alta ajudou a atingir a negatividade do RNA de SARS-CoV-2 em maior proporção, junto com uma diminuição significativa no marcador inflamatório. Isso é importante, pois a negatividade do RNA de SARS-CoV-2 diminui a taxa de transmissão do vírus. Além disso, assim como no estudo de Lakkireddy et al., (2021), não foi relatado efeitos adversos com o uso da alta dosagem, inclusive hipercalemia (Rastogi et $a l ., 2020)$.

Isso mostra que o uso de altas dosagens de vitamina D podem ter uso seguro quando em pacientes com COVID-19 leve ou assintomático e pacientes que não apresentam comorbidades graves, visto que o estudo de Rastogi et al. (2020) e Lakkireddy et al., (2021) excluíram do ensaio pacientes que necessitaram de ventilação invasiva ou com comorbidades significativas. No entanto, doses altas em comparação ao tratamento convencional, exige um acompanhamento rigoroso para verificar a toxicidade da vitamina D, o que limita o seu uso em ambientes hospitalares (Rastogi et al.,2020).

A vitamina D parece regular as respostas imunes inatas e adaptativas, por isso os estudos observacionais mostram que níveis mais elevados de 25-hidroxivitamina D estão associados a melhores resultados clínicos em doenças respiratórias, assim como também foi observada a associação entre níveis baixos de 25-hidroxivitamina D e mau prognóstico entre pacientes com COVID-19 (Carpagnano et al., 2020).

No entanto, no ensaio de Murai et al., (2020), uma dose única de 200.000 UI de vitamina D3 não resultou em nenhum efeito clinicamente relevante entre pacientes hospitalizados com COVID-19 moderado a grave, uma vez que não promoveu mudanças significativas no tempo de internação hospitalar, nem na mortalidade hospitalar e na necessidade de ventilação mecânica., o que contesta o uso de vitamina D3 suplementar como tratamento para pacientes com COVID-19 mais grave.

Por conta disso, os autores desse estudo não apoiam o uso de uma dose elevada de vitamina D3 para o tratamento de COVID-19 moderado a grave de vitamina D, pois apesar de não relatar toxicidade, não gerou mudanças satisfatórias no quadro, apenas atingiu a suficiência de 25-hidroxivitamina D (Murai et al., 2020).

Contudo, esses resultados podem ter sido influenciados pela coexistência de várias doenças nos pacientes, que eram submetidos a um regime medicamentoso diverso, o que poderia ter afetado a homogeneidade da amostra e de seu tratamento. Além disso, os pacientes receberam a dose de vitamina D3 após um período relativamente longo desde o início dos sintomas até a randomização, isto é, uma média de 10,3 dias, o que pode ter influenciado na eficácia da vitamina D (Murai et al., 2020).

O início tardio da suplementação de vitamina D pode não gerar efeitos rápidos em pacientes com COVID-19, uma vez que o quadro inflamatório pode afetar negativamente o metabolismo da $25 \mathrm{OHD}$, principalmente o de sua proteína de ligação, o que acaba resultando em níveis circulantes reduzidos da vitamina e dificultando o aumento dos níveis com a suplementação, o que pode ter acontecido com os pacientes, uma vez que a diferença entre os estudos que trataram pacientes com altas doses de 60.000UI com o estudo de Murai et al., (2020) foi a condição grave dos pacientes (Cereda et al., 2020).

O estudo de Notz et al., (2021) concorda com os resultados do ensaio de Murai et al., (2020), uma vez que os grupos de pacientes dos dois estudos apresentam faixas etárias próximas e são acometidos por COVID-19 no estado grave, sendo os pacientes do ensaio de Notz et al., (2021) acometidos por ARDS. No entanto, nesse estudo foi utilizado uma dose de ataque de 
200.000UI de vitamina D seguida de doses diárias de 10.000UI, que mesmo assim não desencadeou respostas significativas no quadro do paciente e não levou a um aumento geral na 1,25-di-hidroxivitamina D após 10-15 dias.

Os autores explicam esse resultado justificando sobre a falta de um grupo controle no ensaio, o que dificultou na visualização do efeito da vitamina $\mathrm{D}$, além de terem perdido a amostragem durante a fase inicial da doença, visto que a maioria dos pacientes foram encaminhados a outro centro de atendimento (Nortz et al., 2021).

Uma hipótese que também poderia explicar a ineficácia dos bólus de vitamina D na prevenção de infecções agudas do trato respiratório são as flutuações na concentração sérica de $25(\mathrm{OH}) \mathrm{D}$ após a administração em bólus, uma vez que a elevada concentração sérica pode desencadear uma desregulação das enzimas responsáveis pela síntese e degradação do metabolito ativo da vitamina $\mathrm{D}$, isto é, o 1,25 - dihidroxivitamina D, o que resulta em uma menor concentração nos tecidos extra renais, e assim, não promove efeitos significativos no quadro do paciente (Moreira, 2018).

Diante do exposto, é possível observar por meio dos resultados de alguns estudos, como de Rastogi et al., (2020) e Lakkireddy et al., (2021), que a suplementação de vitamina D em pacientes com menos de 65 anos acometidos por COVID-19 leve a moderada e que não foram suplementados com a vitamina antes da doença, apresentaram resultados satisfatórios. No entanto, alguns estudos destacam o impacto positivo da suplementação de vitamina $\mathrm{D}$ antes mesmo do desencadeamento do COVID-19, como o estudo de Annweiler et al., (2020a), Annweiler et al., (2020b) e Annweiler et al., (2021).

Os estudos de mesmo autor, Annweiler et al., (2021) e Annweiler et al., (2020a), foram realizados com pacientes hospitalizados na unidade de cuidados agudos geriátricos do Hospital Universitário de Angers, na França. Ambos demonstraram que o grupo que recebeu suplementação de vitamina $D$ antes do surgimento da doença teve maior tempo de sobrevivência, além da vitamina D em bólus ter sido associada a COVID-19 menos grave em idosos frágeis.

Outro estudo do mesmo autor, Annweiler et al., (2020b), foi realizado com pacientes em uma casa de repouso na França. Nesse ensaio, 82,5\% dos participantes do grupo que recebeu bólus de vitamina D com 80.000UI sobreviveram ao COVID-19, em comparação com apenas 44,4\% no grupo que não recebeu nenhuma suplementação recente.

Um fator que contribuiu para os resultados, foi o fato da suplementação de vitamina D já ser comum em todos os pacientes com mais de 65 anos de idade na França. Isso é importante, pois a suplementação com pequenas doses diárias iniciadas durante COVID-19 é pouco provável que corrija a hipovitaminose D de forma rápida o suficiente para exercer os efeitos imunomoduladores e melhorar o prognóstico da infecção, em comparação com a suplementação por bólus de vitamina D administrados antes ou no início da doença, pois apresentam maior probabilidade de aumentar rapidamente a concentração de 25 (OH) D e melhorar o prognóstico de COVID-19 em comparação com as doses diárias (Bruyere et al., 2015).

Por fim, o único estudo que relatou risco da suplementação de vitamina D em pacientes com COVID-19 foi o de Cereda et al., (2021), em que foi estudado dois conjuntos de dados, o primeiro consistia em pacientes e cuidadores com COVID-19 que foram abordados por telefone, e o segundo foi composto por pacientes COVID-19 internados em um hospital. Nesse estudo, foi encontrada uma tendência para uma mortalidade duas vezes maior em pacientes que fizeram suplementação.

No entanto, os autores explicam essa controvérsia em relação a literatura justificando sobre o fato de a inflamação afetar negativamente o metabolismo da 25OHD, o que pode ter desencadeado o resultado diferente da literatura. Além disso, muitos dados como dosagem e tempo de intervenção não foram relatados, assim como a dosagem de vitamina D não foi padronizada entre os participantes, o que dificulta um resultado concreto sobre a suplementação.

Diante do exposto, destaca-se o potencial da suplementação prévia de vitamina D para um bom prognóstico no COVID19, principalmente em pacientes geriátricos ou que apresentam comorbidades, como diabetes hipertensão, doenças cardíacas, que são grupos que já apresentam uma deficiência de vitamina $\mathrm{D}$, pois reduz o risco de infecções agudas do trato respiratório (Mohammad et al., 2019). No entanto, os efeitos sobre a imunidade inata após a exposição ao vírus respiratório são limitados e 
seu uso em várias concentrações pode não inibir as respostas pró inflamatórias, o que justifica os resultados insignificantes dos estudos com pacientes com COVID-19 grave (Fitch et al., 2016).

Diante dos resultados estudados, é notória a relevância da suplementação de vitamina D diária ou reposição alimentar, uma vez que a tendência é a convivência nos próximos anos com o vírus que acomete o mundo atualmente. Por isso, é importante que os profissionais de saúde, principalmente o nutricionista, atue diretamente na inserção da vitamina na dieta da população para atingir um nível saudável de vitamina D (Manson et al., 2020).

A vitamina D é encontrada em alimentos, como por exemplo, salmão, cogumelos, sardinhas, ovos, iogurte, fígado de boi e ostras, além de também ser produzida pelo sistema endógeno, quando os raios ultravioletas da luz solar atingem a pele e desencadeiam a síntese da vitamina D. Embora pesquisas adicionais sobre a contribuição de vitamina D para atenuar o risco e a gravidade do COVID-19 estejam em andamento, a diminuição da sua deficiência melhorará a saúde pública durante a atual pandemia, assim como nos próximos anos, evitando picos de infecção na população do país (Oliveira et al., 2020).

Dessa forma, uma alimentação saudável e adequada está associada ao fortalecimento do sistema imunológico, o que representa um aspecto fundamental no enfrentamento da COVID-19, requerendo dos profissionais nutricionistas a realização de intervenções, por meio de prescrições dietéticas e orientações sobre alimentação saudável na perspectiva de prevenção, tratamento e reabilitação de pessoas acometidas por essa enfermidade (Dutra et al., 2020).

\section{Considerações Finais}

Diante do exposto, foi possível concluir pelas evidências encontradas que a suplementação de vitamina D é mais eficaz em pacientes com COVID-19 de leve a moderada, principalmente em pacientes que já faziam suplementação da vitamina antes de serem acometidos pela doença. Sobre os pacientes com COVID-19 grave, as evidências mostraram que altas doses podem gerar efeitos rápidos e satisfatórios no tempo de internação, porém o início tardio da suplementação pode ser um fator limitador.

Com os resultados, é possível destacar também a importância da realização de mais estudos no futuro sobre o uso da vitamina D e seus efeitos em pacientes acometidos por COVID-19, a fim de se definir uma dosagem que se mostre eficaz na prevenção da doença, assim como também para ser usada como tratamento auxiliar.

\section{Referências}

Albuquerque, A. B. B. (2021). Vitamina D em pacientes com COVID-19: atuais perspectivas. 2021. 34f. Trabalho de Conclusão de Curso, Departamento de Nutrição, Universidade Federal do Rio Grande do Norte, Natal, Rio Grande do Norte, Brasil.

Annweiler, G., Corvaisier, M., Gautier, J., Dubée, V., Legrand, E., Sacco, G., \& Annweiler, C. (2020). Vitamin D Supplementation Associated to Better Survival in Hospitalized Frail Elderly COVID-19 Patients: The GERIA-COVID Quasi-Experimental Study. Nutrients, 12(11), 3377. https://doi.org/10.3390/nu12113377

Annweiler, C., Beaudenon, M., Simon, R., Guenet, M., Otekpo, M., Célarier, T., \& Gautier, J. (2021). Vitamin D supplementation prior to or during COVID-19 associated with better 3-month survival in geriatric patients: Extension phase of the GERIA-COVID study. The Journal of Steroid Biochemistry and Molecular Biology, 213, 105958. https://doi.org/10.1016/j.jsbmb.2021.105958

Annweiler, C., Hanotte, B., Grandin de l'Eprevier, C., Sabatier, J.-M., Lafaie, L., \& Célarier, T. (2020). Vitamin D and survival in COVID-19 patients: A quasiexperimental study. The Journal of Steroid Biochemistry and Molecular Biology, 204, 105771. https://doi.org/10.1016/j.jsbmb.2020.105771

Bleizgys, A. (2020). Vitamin D and COVID-19: It is time to act. International Journal of Clinical Practice, 75(3). https://doi.org/10.1111/ijcp.13748

Bruyère, O., Deroisy, R., Dardenne, N., Cavalier, E., Coffiner, M., Da Silva, S., De Niet, S., \& Reginster, J.-Y. (2015). A phase IV, two-armed, randomized, cross-over study comparing compliance with once-a-month administration of vitamin D3 to compliance with daily administration of a fixed-dose combination of vitamin D3 and calcium during two 6-month periods. Osteoporosis International, 26(12), 2863-2868. https://doi.org/10.1007/s00198-015-3205-z

Cereda, E., Bogliolo, L., de Stefano, L., \& Caccialanza, R. (2020). A brief discussion of the benefit and mechanism of vitamin D supplementation on coronavirus disease 2019. Current Opinion in Clinical Nutrition \& Metabolic Care, 24(1), 102-107. https://doi.org/10.1097/mco.0000000000000701

Carpagnano, G. E., Di Lecce, V., Quaranta, V. N., Zito, A., Buonamico, E., Capozza, E., Palumbo, A., Di Gioia, G., Valerio, V. N., \& Resta, O. (2020). Vitamin $\mathrm{D}$ deficiency as a predictor of poor prognosis in patients with acute respiratory failure due to COVID-19. Journal of Endocrinological Investigation, 44(4), 765771. https://doi.org/10.1007/s40618-020-01370-x 
Research, Society and Development, v. 10, n. 14, e485101422188, 2021

(CC BY 4.0) | ISSN 2525-3409 | DOI: http://dx.doi.org/10.33448/rsd-v10i14.22188

Dutra, A. de F. de F. de O., Dias, A. D. C., Araújo, D. G. de S., Silva, E. M. da, Silva, I. M. F. e, \& Gomes, L. M. de F. (2020). A importância da alimentação saudável e estado nutricional adequado frente a pandemia de Covid-19. Brazilian Journal of Development,6(9), 66464-66473. https://doi.org/10.34117/bjdv6n9-181

Estrela, C. (2018). Metodologia Científica: Ciência, Ensino, Pesquisa. Editora Artes Médicas.

Fitch, N., Becker, A. B., \& HayGlass, K. T. (2016). Vitamin D [1,25(OH)2D3] Differentially Regulates Human Innate Cytokine Responses to Bacterial versus Viral Pattern Recognition Receptor Stimuli. The Journal of Immunology, 196(7), 2965-2972. https://doi.org/10.4049/jimmunol.1500460

Lakkireddy, M., Gadiga, S. G., Malathi, R. D., Karra, M. L., Raju, I. S. S. V. P. M., Ragini, ... Kandakatla, M. (2021). Impact of daily high dose oral vitamin D therapy on the inflammatory markers in patients with COVID-19 disease. Scientific Reports, 11(1). https://doi.org/10.1038/s41598-021-90189-4

Manson, J. E., \& Bassuk, S. S. (2020). Commentary. Eliminating vitamin D deficiency during the COVID-19 pandemic: A call to action. Metabolism, 112 154322. https://doi.org/10.1016/j.metabol.2020.154322

Moreira, V. (2018). Vitamina D, a nova arma contra as infeções respiratórias agudas. Revista Portuguesa de Medicina Geral E Familiar, 34(1), 48-50. https://doi.org/10.32385/rpmgf.v34i1.12364

Moreira, T. P., Costa, V. de S., Ferreto, L. E. D., \& Wendt, G. W. (2020). Efeitos da vitamina D na prevenção e tratamento da COVID-19: uma revisão sistemática. Research, Society and Development, 9(12). https://doi.org/10.33448/rsd-v9i12.11174

Murai, I. H., Fernandes, A. L., Sales, L. P., Pinto, A. J., Goessler, K. F., Duran, C. S. C., Pereira, R. M. R. (2021). Effect of a Single High Dose of Vitamin D3 on Hospital Length of Stay in Patients With Moderate to Severe COVID-19. JAMA, 325(11), 1053. https://doi.org/10.1001/jama.2020.26848.

Notz, Q., Herrmann, J., Schlesinger, T., Kranke, P., Sitter, M., Helmer, P., Stumpner, J., Roeder, D., Amrein, K., Stoppe, C., Lotz, C., \& Meybohm, P. (2021). Vitamin D deficiency in critically ill COVID-19 ARDS patients. Clinical Nutrition, 21. https://doi.org/10.1016/j.clnu.2021.03.001

Ohaegbulam, K. C., Swalih, M., Patel, P., Smith, M. A., \& Perrin, R. (2020). Vitamin D Supplementation in COVID-19 Patients: A Clinical Case Series. American Journal of Therapeutics, 27(5), 485-490. https://doi.org/10.1097/mjt.0000000000001222

Oliveira, E. de S., Matos, M. F., Cavalcante, O. S. S., Silvestre, J. V. C., Souza, D. E. M. de, \& Morais, A. C. L. N. de. (2020). As duas faces da vitamina D como terapia adjuvante na covid-19. InterAmerican Journal of Medicine and Health, 3. https://doi.org/10.31005/iajmh.v3i0.95

Rastogi, A., Bhansali, A., Khare, N., Suri, V., Yaddanapudi, N., Sachdeva, N., \& Malhotra, P. (2020). Short term, high-dose vitamin D supplementation for COVID-19 disease: a randomised, placebo-controlled, study (SHADE study). Postgraduate Medical Journal, 1-4. https://doi.org/10.1136/postgradmedj-2020139065

Rizzoli, R. (2020). Vitamin D supplementation: upper limit for safety revisited? Aging Clinical and Experimental Research, 33(1), 19-24. https://doi.org/10.1007/s40520-020-01678-x

Sabico, S., Enani, M. A., Sheshah, E., Aljohani, N. J., Aldisi, D. A., Alotaibi, N. H., \& Al-Daghri, N. M. (2021). Effects of a 2-Week 5000 IU versus 1000 IU Vitamin D3 Supplementation on Recovery of Symptoms in Patients with Mild to Moderate Covid-19: A Randomized Clinical Trial. Nutrients, 13(7), 2170. https://doi.org/10.3390/nu13072170 